\title{
Cardiovascular disease during the COVID-19 pandemic: Think ahead, protect hearts, reduce mortality
}

\author{
Guoliang $\mathrm{Li}^{1,2,3 *}$, Ardan M. Saguner ${ }^{4 *}$, Jiaqi $\mathrm{An}^{2,5,6 *}$, Yuye Ning ${ }^{1,2,5}$, \\ John D. Day ${ }^{7}$, Ligang Ding ${ }^{8}$, Xavier Waintraub ${ }^{3}$, Jie Wang ${ }^{1,9}$
}

${ }^{1}$ Department of Cardiovascular Medicine, The First Affiliated Hospital of Xi'an Jiaotong University, Xi'an, China ${ }^{2}$ Atrial Fibrillation Center, The First Affiliated Hospital of Xi'an Jiaotong University, Xi'an, China

${ }^{3}$ Cardiology Institute, Rhythmology Unit, Pitié-Salpêtrière Hospital, Paris, France

${ }^{4}$ Department of Cardiology, University Heart Center Zurich, Switzerland

${ }^{5}$ Stroke Center and Department of Neurology, The First Affiliated Hospital

of Xi'an Jiaotong University, Xi'an, China

${ }^{6}$ Department of Neurology, Massachusetts General Hospital, Boston, MA, United States

${ }^{7}$ Intermountain Medical Center Heart Institute, Murray, UT, United States

${ }^{8}$ Department of Cardiovascular Medicine, Clinical EP Lab and Arrhythmia Center, Fuwai Hospital,

State Key Laboratory of Cardiovascular Disease, National Center for Cardiovascular Diseases,

Chinese Academy of Medical Sciences and Peking Union Medical College, Beijing, China

${ }^{9}$ Department of Hematology, The First Affiliated Hospital of Xi'an Jiaotong University, Xi'an, China

\begin{abstract}
Coronavirus disease 2019 (COVID-19) is rapidly spreading globally. As of October 3, 2020, the number of confirmed cases has been nearly 34 million with more than 1 million fatalities. Severe acute respiratory syndrome coronavirus 2 (SARS-CoV-2) is accountable for COVID-19. Newly diagnosed and worsening cardiovascular disease are common complications in COVID-19 patients, including acute cardiac injury, hypertension, arrhythmia, myocardial infarction, heart failure and sudden cardiac arrest. The mechanisms contributing to cardiac disease burden include hypoxemia, inflammatory factor storm, dysfunctional angiotensin converting enzyme 2 (ACE2), and drug-induced cardiac toxicity. Notably, the macrophages expressing ACE2 as direct host cells of SARS-CoV-2 secrete chemokine and inflammatory cytokines, as well as a decrease in cellular immune responses to SARS-CoV-2 infection due to elevated exhaustion levels and dysfunctional diversity of $T$ cells, that may be accountable for the "hyperinflammation and cytokine storm syndrome" and subsequently acute cardiac injury and deteriorating cardiovascular disease in COVID-19 patients. However, no targeted medication or vaccines for COVID-19 are yet available. The management of cardiovascular disease in patients with COVID-19 include general supportive treatment, circulatory support, other symptomatic treatment, psychological assistance as well as online consultation. Further work should be concentrated on better understanding the pathogenesis of COVID-19 and accelerating the development of drugs and vaccines to reduce the cardiac disease burden and promote the management of COVID-19 patients, especially those with a severe disease course and cardiovascular complications. (Cardiol J 2020; 27, 5: 616-624)
\end{abstract}

Key words: COVID-19, angiotensin converting enzyme 2, cardiovascular complications, inflammatory factor storm, endotheliitis, online consultation

Address for correspondence: Guoliang Li and Jie Wang, Department of Cardiovascular Medicine, The First Affiliated Hospital of Xi'an Jiaotong University, No. 277 Yanta West Road, Xi'an 710061, China, tel: 86-02985323805, fax: 86-02985323805, e-mail: liguoliang_med@163.com and wj-8871@163.com

Received: 30.04.2020 Accepted: 21.07.2020

*These authors contributed equally to this work and should be considered to share first authorship. 


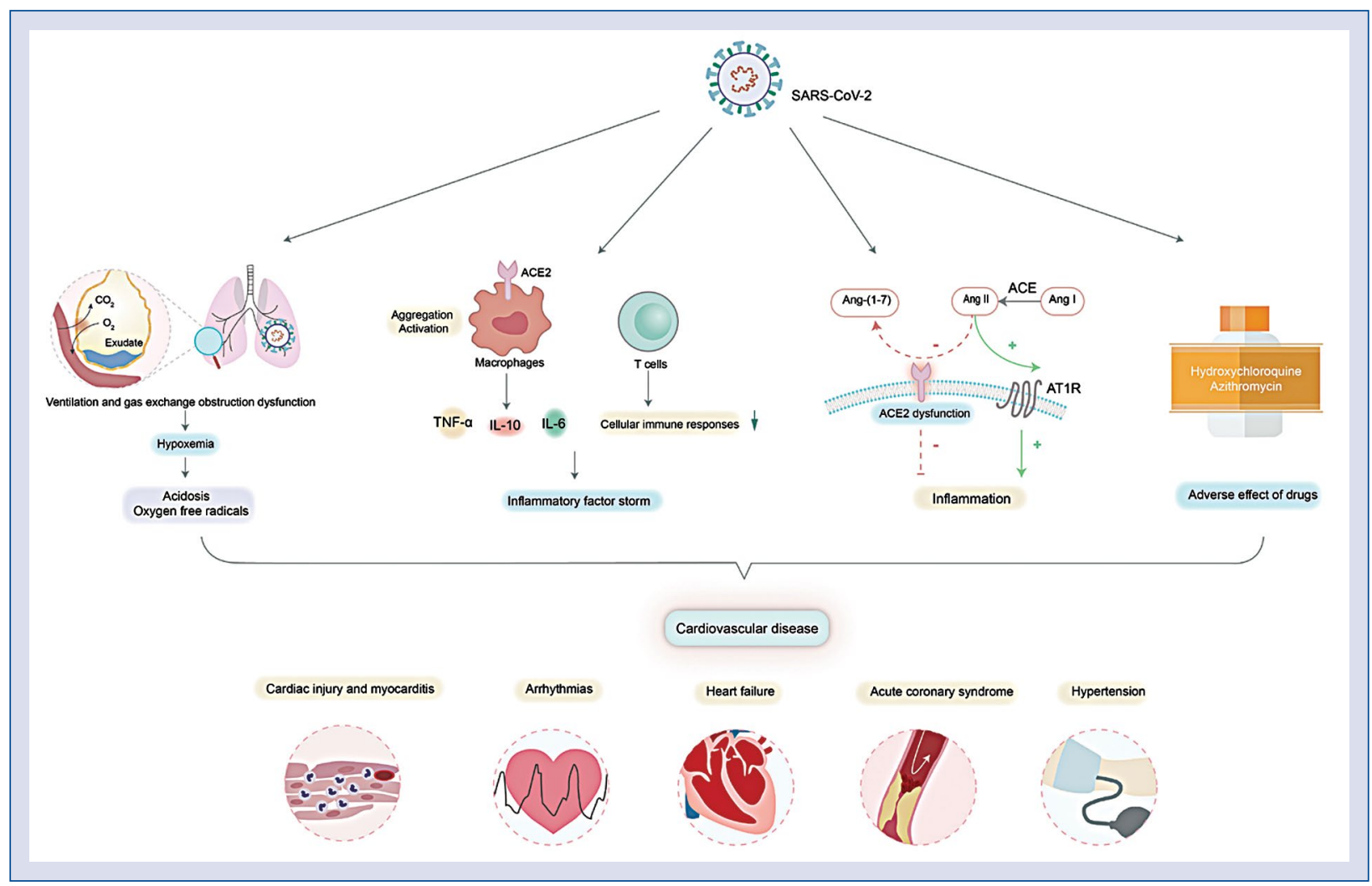

Figure 1. Coronavirus disease 2019 (COVID-19) and cardiovascular disease; SARS-CoV-2 — severe acute respiratory syndrome coronavirus 2; ACE2 - angiotensin-converting enzyme 2; Ang — angiotensin; AT1R - angiotensin II type 1 receptor; TNF- $\alpha$ — tumor necrosis factor alpha; IL-10 — interleukin-10; IL-6 — interleukin-6.

\section{Introduction}

Coronavirus disease 2019 (COVID-19) is rapidly spreading globally. As of October 3, 2020, the number of confirmed cases has been nearly 34 million with more than 1 million fatalities. Severe acute respiratory syndrome coronavirus 2 (SARS-CoV-2) is accountable for COVID-19 and its sequence analysis has the smallest genetic distance from bat coronavirus and shares a $79.5 \%$ sequence identity to SARS-CoV [1]. SARS-CoV-2 has a much more efficient transmission through active pharyngeal viral shedding at the time when symptoms are still mild and typical of upper respiratory tract infection, and spreads much faster than SARS-CoV [2]. SARS-CoV-2 can infect humans by gaining $\mathrm{S}$ protein-driven viral entry to a cell by utilizing angiotensin converting enzyme 2 (ACE2) [3, 4].

Patients with COVID-19 have multiple organ system dysfunction [5-7], and this was further confirmed by the latest pathological findings from systematic autopsy (37 cases) and percutaneous multiple organ biopsy (54 cases) [8]. According to the largest study to date [9] and recent data from other studies [5, 6, 8, 10-12], acute cardiac injury and underlying cardiovascular disease (CVD) are common in patients with COVID-19. On the other hand, patients with cardiovascular comorbidities are more prone to suffer from COVID-19, which in turn can cause deterioration of their CVD. This work reviewed the cardiovascular complications in COVID-19 patients, the underlying mechanisms, the management, and the prospect and challenges, aiming to reduce the cardiac disease burden and promote the management of COVID-19 patients, especially those with a severe disease course and cardiovascular complications (Fig. 1).

\section{COVID-19 and CVD}

\section{Cardiac injury and myocarditis}

Abnormal elevation of cardiac injury biomarkers is widely present in patients with COVID-19 and is likely associated with infection-related myocarditis, right heart strain, and/or ischemia [10]. As for outcomes, the cardiac injury bio-markers are closely related to the disease progression and prognosis [10]. In a cohort study of patients 
with COVID-19, cardiac injury occurred in $19.7 \%$ of patients during hospitalization, and it was an independent risk factor for in-hospital mortality [10]. Cardiac injury was diagnosed mainly by an increased level of high-sensitivity cardiac troponin I (hs-cTnI). Patients with cardiac injury had a higher prevalence of chronic disease, including hypertension, diabetes, coronary artery disease, cerebrovascular disease, chronic heart failure (HF), and cancer. As for clinical outcome, cases with cardiac injury had much higher fatality rates $(51.2 \%)$ than those without cardiac injury $(4.5 \%)$. In a retrospective and multicenter cohort study of 191 patients with COVID-19 from hospitals in Wuhan, China, acute cardiac injury was observed in $17 \%$ of all cases, $59 \%$ of non-survivors and $1.0 \%$ of survivors. Hs-cTnI were $22.2 \mathrm{pg} / \mathrm{mL}$ and $3.0 \mathrm{pg} / \mathrm{mL}$ in non-survivors and survivors, respectively. In addition, levels of hs-c TnI were elevated in non-survivors compared with survivors throughout the clinical course, and increased with illness deterioration [6]. Taken together, these findings are supportive of the idea that myocardial injury is closely related to the severity and prognosis of patients with COVID-19.

SARS-CoV-2 can cause myocarditis [13-15]. In a case report of a patient with the clinical presentation of myocarditis, the cardiac function sharply decreased and the heart size showed significant enlargement with troponin $\mathrm{T}$ of more than $10,000 \mathrm{ng} / \mathrm{L}$, creatinine kinase-MB $112.9 \mathrm{ng} / \mathrm{L}$ and B-type natriuretic peptide (BNP) was up to $21,025 \mathrm{ng} / \mathrm{L}$ [15]. Notably, there is no clear evidence that SARS-CoV-2 can directly impair the myocardium and cause viral myocarditis, because neither SARS-CoV-2 in myocardial tissue nor substantial damage were detected in heart tissue obtained from postmortem biopsies $[13,16]$. The most recent pathological evidence from deceased patients undergoing necropsy indicated no viral particles in cardiac parenchymal cells, which is supportive of previous findings [17]. Thus, it appears that myocarditis is likely secondary to an inflammatory storm, right heart strain and/or ischemia.

\section{Arrhythmias in COVID-19}

Electrocardiogram abnormalities and arrhythmias were observed in more than $70 \%$ of patients with SARS and are also common in patients with COVID-19 [18-21]. More than 74\% of patients with COVID-19 showed electrocardiogram abnormalities and arrhythmias [22].

Sinus tachycardia is commonly observed in COVID-19 patients reporting palpitations, whereas atrial tachycardia and atrial fibrillation are common in patients with symptoms of fatigue and chest tightness. Ventricular premature beats and paroxysmal ventricular tachycardia (VT) are common in patients with palpitations, dizziness, and even syncope. In severe cases, patients suffering from an inflammatory storm are at high risk of sustained VT and even ventricular fibrillation [22]. Patients with COVID-19 who develop cardiac injury often present with sinus tachycardia [19]. Bradyarrhythmias are infrequent in patients with COVID-19, but they seem to occur suddenly and most of these cases are due to high-level atrioventricular block [22]. The QTc interval was $431 \mathrm{~ms}$ in patients with COVID-19 and $12.9 \%$ of cases showed prolonged QTc interval [22]. Hydroxychloroquine and azithromycin, which are used as off-label drugs to treat COVID-19 in some areas, can further enhance QT prolongation and may predispose patients with and without underlying CVDs to potentially lifethreatening torsade de pointes VT [23-25].

The abnormal electrocardiogram findings were related to the underlying severity of COVID-19 patients. In a case series of COVID-19 patients with ST-segment elevation, $72 \%$ of cases died in the hospital [20]. Compared with the non-invasive care unit (ICU) group, the proportion of abnormal $\mathrm{Q}$ waves was higher in the ICU group $(33.3 \%$ vs. 3.9\%) [22]. Abnormal $Q$ waves often indicate myocardial necrosis. Viral infection can aggravate the original myocardial disease and increase the risk of critical illness through mechanisms such as immune damage, aggravation of microvascular ischemia and induction of apoptosis.

\section{Heart failure and sudden cardiac arrest}

Heart failure is common in cases with COVID-19 [5, 26]. In a retrospective study of 112 cases with COVID-19 divided into non-survivors $(17,15.18 \%)$ and survivors $(95,84.82 \%)$, there were $27(28.42 \%)$ and $13(76.47 \%)$ cases with HF, respectively. The most recent report to date from Italy indicated that the second most common comorbidity in patients with COVID-19 admitted to ICUs were cardiomyopathy and HF. This finding is also supportive of the idea that HF may predispose individuals with COVID-19 to a worsening clinical outcome [5]. Even among COVID-19 patients without chronic underlying disease, those patients developing acute $\mathrm{HF}$ within a short time are prone to sudden cardiac arrest and the course of the disease often deteriorates rapidly [18].

Heart failure in cases with COVID-19 appears secondary to acute respiratory distress syndrome 
(ARDS), acute respiratory failure, or other serious respiratory complications [18]. Pulmonary artery hypertension and pulmonary heart disease due to increased pulmonary vascular resistance leads to increased right ventricular strain and right HF. One should be alert to HF to reduce mortality. Moreover, COVID-19 patients at risk often have diastolic dysfunction and/or microvascular disease with increased left-sided filling pressures, which in case of a superinfection and fluid overload, can aggravate left-HF and increase pulmonary edema thereby deteriorating ARDS.

\section{Acute coronary syndrome}

Acute coronary syndrome (ACS) ranks second after respiratory failure as the cause of fatality [22, 26], and most cases in patients with COVID-19 are secondary to severe respiratory failure. Lactic acid accumulation and hypoxia caused by respiratory failure and hypercoagulation accelerates the occurrence of acute myocardial infarction (AMI), especially in cases with underlying CVD. Emergency percutaneous coronary intervention (PCI) should be considered to improve the ischemia-reperfusion injury. Given the lack of negative pressure cardiac catheterization chambers during the COVID-19 pandemic, particularly in regions with less advanced health-care systems, $\mathrm{PCI}$ as an emergency procedure has to be reconsidered, particularly for non-ST-segment elevation myocardial infarction, and prehospital emergency intravenous thrombolytic therapy is generally recommended. Expert consensus and guidelines on the management of ACS emphasizing the emerging role of intravenous thrombolytic therapy during the COVID-19 pandemic have been presented by scientific societies or groups on the front line against COVID-19 [27-29].

\section{Hypertension}

Half of cases with COVID-19 have comorbidities and the most common one is systemic arterial hypertension, which is associated with the severity of COVID-19 and in-hospital death $[6,7]$. In a retrospective cohort study, $30 \%$ of cases had hypertension and the prevalence of hypertension in non-survivors (48\%) was twice as much as that in non-survivors (23\%) [6]. In a recent study, hypertension was the most common comorbidity (49\%) in patients with laboratory-confirmed COVID-19 referred for ICU admission in the Lombardy region of Italy [5]. In a study by Huang et al. [7], 15\% of cases have hypertension and the ICU group had higher systolic blood pressure as compared to the non-ICU group.

\section{Mechanisms of CVD in COVID-19}

At present, the mechanisms underlying cardiac injury and other CVDs in patients with COVID-19 are not well defined. Possible mechanisms are the following: an excessive inflammatory reaction, severe hypoxemia, dysfunctional ACE2 with upregulation of the renin-angiotensin system, cardiac toxicity due to drug-drug interactions such as azithromycin and hydrochloroquine causing acquired long QT syndrome with torsade de pointes ventricular tachycardia.

\section{Hypoxemia}

Respiratory infection leading to insufficient oxygen supply is the main cause of hypoxemia $[5,6,17]$. In the most recent work by Wang et al. [17], pathologic investigations of fatal cases undergoing necropsy provide new evidence contributing to the severe dysfunction of ventilation and gas exchange obstruction in patients with COVID-19. The gross anatomy of the lungs showed moderate bilateral pleural effusion and pleural adhesion in 2 patients and hepatization of lung tissue was observed. As for microscopic manifestation, a massive serous and fibrinoid exudate was observed in the alveolar spaces [17]. On the other hand, infection-induced increased metabolic requirements need an enhanced oxygen supply, which in turn results in severe hypoxemia. And hypoxemia, in turn, may lead to increased anaerobic fermentation and subsequently acidosis, oxygen free radicals, and ultimately cardiac injury. Among COVID-19 patients with underlying CVD the risk of hypoxemia is increased. And this hypoxemia can aggravate cardiac injury and accelerate the progression of CVD. These findings may explain the high rate of $\mathrm{ACS}$, acute $\mathrm{HF}$ and fatal arrhythmias in severe cases. Notably, emerging pathological evidence from post mortem examination indicates pulmonary hemorrhage which may also be accountable for hypoxemia induced by suboptimal ventilation [17]. However, the causes of hypoxemia and its impact on the progression of ARDS needs to be further elucidated.

\section{Inflammatory factor storm}

Cytokine storm plays a vital role in the pathogenesis of coronavirus-caused tissue damage and entails a vast amount of cardiac injury in patients infected by coronaviruses $[6,7,17,30]$. As for patients with COVID-19, the level of interleukin-6 
(IL-6) was higher in non-survivors than in survivors throughout the clinical course, and increased with illness deterioration [6]. In Huang's study, higher levels of plasma cytokines and chemokines were higher in ICU patients than non-ICU patients [7].

The most recent evidence provides novel contributions for a better understanding of the immune responses during COVID-19 [17, 30, 31]. First, pathological evidence obtained from fatal cases due to COVID-19 indicates that a direct viral infection of the macrophages expressing ACE2 results in the extraordinary aggregation and activation of macrophages. These macrophages infected by SARS-CoV-2 secrete chemokines and inflammatory cytokines including IL-6, IL-10 and tumor necrosis factor alpha $(\mathrm{TNF} \alpha)$. These findings are supportive of the idea that macrophages act as direct host cells of SARS-CoV-2 and potential drivers of "inflammatory factor storm" or "cytokine storm" in COVID-19 [17]. On the other hand, decreased cellular immune responses to SARS-CoV-2 infection were also identified. Elevated exhaustion levels and dysfunctional diversity of $\mathrm{T}$ cells may be another mechanism accountable for the "cytokine storm syndrome" in COVID-19 [30]. Previous studies indicated that multi-functional T cells can better control human immunodeficiency virus and are correlated with better outcomes during vaccination. However, in Zhang and colleagues' study [30], SARS-CoV-2 infection caused dysfunctional CD4+ T cells and promoted extraordinary activation and possibly subsequent exhaustion of $\mathrm{CD} 8+\mathrm{T}$ cells. Moreover, severe cases infected by SARS-CoV-2 showed significantly decreased frequency of multi-functional CD4+ T cells compared with healthy controls and mild cases. These findings are supportive of the idea that compared to other coronaviruses, SARS-CoV-2 may possess a unique immunopathological mechanism which predisposes infected cases to deteriorate rapidly.

\section{Dysfunctional ACE2}

Previous data indicated that coronavirus can lead to ACE2 dysfunction and subsequently abnormal activation of renin-angiotensin system, eventually resulting in an hyperinflammatory reaction. The down-regulation of ACE2 was observed in animal models of SARS and H7N9 infection, and this can be counter regulated by angiotensin receptor blockers (ARBs) [32, 33]. As for the cardiovascular system, the decreased ACE2 expression in the myocardium was observed in animals and patients infected by SARS-CoV [34]. Infection with the human SARS-CoV in mice led to an ACE2-dependent myocardial infection with a marked decrease in ACE2 expression, which was supportive of a critical role of ACE2 in mediating SARS-CoV infection in the heart. In patients infected by SARS-CoV, the virus was also detected in autopsied human hearts and its presence was associated with marked reductions in ACE2 protein expression [34]. Though no evidence of SARS-CoV-2 directly infecting myocardium, based on the information that SARS-CoV-2 and SARS-CoV share the similar structure and function $[4,35]$. We can make some rational and scientific inferences that SARS-CoV-2 may lead to cardiac injury by affecting ACE2 function. Notably, emerging evidence from post-mortem analysis are supportive of this idea [36]. In a serial section of tissues, Varga et al. [36] found evidence of direct SARS-CoV-2 infection of the endothelial cell and diffuse endothelial inflammation. The fact that endothelial cells of multiple systems expressing dysfunctional ACE2 receptor due to direct SARS-CoV-2 infection of the endothelium or immune-mediated facilitates the induction of endotheliitis and may be the main mechanism underlying multiple system organ failure, including CVD [36]. Drugs or vaccines targeting these processes may provide a therapeutic target.

\section{Adverse effect of drugs}

Given the fast spread and high transmission rates of COVID-19, there are many programs aiming at developing pharmaceutical drugs for treatment and prevention of COVID-19. While ongoing and future studies should be actively supported, caution is needed for the off-label use of previously approved drugs due to cardiac toxicity, especially in severe cases with multiple comorbidities and polypharmacy [5-7, 21]. In a small study conducted by Gautret et al. [37] attracting much attention, hydroxychloroquine treatment was significantly associated with viral load reduction/disappearance in the pharynx of COVID-19 patients. In China, hydroxychloroquine is already recommended in the Chinese Clinical Guidance for COVID-19 Pneumonia Diagnosis and Treatment. Other countries have followed these recommendations. However, clinical and in vitro experimental evidence has reported hydroxychloroquine-induced cardiac toxicity [21, 38 ]. When hydroxychloroquine was prescribed in severe cases, patients with underlying CVD are prone to hydroxychloroquine-induced cardiac toxicity and this is likely to aggravate underlying disease. In a recent study, patients with COVID-19 treated with hydroxychloroquine and azithromycin had severe QTc interval prolongation but they had 
a normal QTc at baseline [21]. Another factor should also be considered, namely synergistic toxicity. Patients with COVID-19 are often concurrently prescribed a drug in combination with many other drugs.

\section{Management of CVD during the COVID-19 pandemic}

In the management of COVID-19, the treatment of patients with acute myocardial injury and underlying CVD adheres to the principles of comprehensive treatment. In addition to the general and supportive treatment, antiviral therapy is recommended in expert consensus and guidelines. However, there are no targeted antiviral agents for SARS-CoV-2. Though lopinavir/ritonavir and hydroxychloroquine showed effectiveness in some cases, recent evidences from a randomized, controlled, open-label trial published in 'New England Journal of Medicine' [39] showed that no benefit was observed with lopinavir/ritonavir treatment beyond standard care in hospitalized adult patients with severe COVID-19. As for chloroquine, the article by Raoult [40] to support the potential of the chloroquine has been retracted due to "expected standard". In addition, the same dosing regimen reported by Molina et al. [41] showed no evidence of a strong antiviral activity or clinical benefit of the combination of hydroxychloroquine and azithromycin for the treatment of the hospitalized patients with severe COVID-19. Thus, the therapeutic value and safety of these antiviral agents are still under investigation with ongoing trials.

\section{Respiratory support}

Depending on the clinical condition, supplemental oxygen and invasive respiratory support should be considered as expert consensus guidelines recommend. However, more awareness is needed when ICU capacity is overwhelmed. Viral transmission through exhaled air dispersion during respiratory support can accelerate interpersonal transmission in the airtight wards, and this situation may be worse when two or more patients are using a shared ventilator due to limited heath care resources [42].

\section{Anti-inflammatory treatment}

As for inflammatory storm, the administration of immunoglobulins can effectively ameliorate the strong immune response to SARS-CoV-2. Recent pathological evidence from post mortem examinations indicated characteristic abnormalities of the mucous plug with fibrinous exudate in the alveoli and the activation of alveolar macrophages [17]. These findings are supportive of the potential of the IL-6 receptor antagonist tocilizumab for severe and critically ill patients with COVID-19. The use of glucocorticoids is controversial and currently not recommended in the current interim guidance from the World Health Organization on clinical management of COVID-19 due to its potential inhibition of viral clearance and prolongation of the duration of viremia [43]. Both artificial liver support systems and continuous renal replacement therapy showed potential in the treatment of SARS and MERS, and are also recommended in the guidelines of the Chinese National Health Commission against COVID-19.

\section{Drug treatment}

As for those with CVD, statins, $\beta$-blockers, ACEIs/ARBs, and antiplatelet and anticoagulant agents should be actively prescribed as appropriate to protect the cardiovascular system. As for AMI, emergency PCI should be the first choice if a negative pressure catherization room is available. The strategies to treat arrhythmias in patients with COVID-19 should be according to the type of arrhythmia and the hemodynamic status of the patient.

Most notably, the use of ACEIs/ARBs in patients without CVD are not recommended. Whether ACEIs/ARBs are beneficial in COVID-19 patients without hypertension, HF, ischemic cardiomyopathy or other diseases are still under investigation.

\section{Device treatment}

Regarding cardiac pump failure, life supportive treatments such as a temporary pacemaker, left ventricular assist device, and extracorporeal membrane oxygenation (ECMO) should also be considered early in the disease course. Given that severe or critical COVID-19 cases who can be successfully weaned off ECMO have been reported to be rare, some have argued that ECMO might not be an optimal treatment strategy during the COVID-19 pandemic. Herein provided is 1 case supportive of ECMO as a life-saving procedure to provide both respiratory and cardiac support for patients suffering from cardiac and respiratory failure. This case is a patient with laboratory-confirmed COVID-19. Her condition rapidly deteriorated under conventional therapy. VV-ECMO was used to provide both respiratory and cardiac support. She had fully recovered from the condition that necessitated the use of ECMO after about 7 weeks. The ECMO treatment in this case is supportive of the use of ECMO during the COVID-19 pandemic. 


\section{Psychological assistance}

The relationship of mental disorders and CVD is well known. Mental health disorders, such as anxiety, fear, depression, and insomnia are very common in patients, health professionals, and the general public [44, 45]. Governments, organizations, and institutions have implemented policies to improve these mental health challenges. Though the potential of mental health disorders to aggravate underlying CVD or induce acute cardiac injury during the COVID-19 pandemic remains unknown, psychological assistance should be provided as soon as possible by a hotline or online consultation [44-46].

\section{Online consultation}

Given that COVID-19 has overwhelmed many healthcare systems, non-infectious disease, intensivists, and cardiologist physicians have been recruited from other medical specialties, without adequate training, to play this role. During the COVID-19 pandemic in China, specialists in other cities were invited for online consultation for the management of severe and critical cases in hospitals. When COVID-19 was rapidly spreading all over the world in March of 2020, hospitals in China also shared clinical experiences with specialists in Italy and the United States. Fast and effective online consultation are important when the appropriate specialists are not available. Social distancing however does not mean social isolation. We should all stand together against the COVID-19 pandemic globally with the help of an online consultation system.

Last but importantly, thinking ahead is a precondition for protecting hearts and reducing mortality. We must bear in mind the clinical presentation and other clues of myocardial injury in this challenging disease, and carefully monitor cardiac function and myocardial injury. Based on our experience in Wuhan hospitals, the typical clinical manifestation with angina pectoris, shortness of breath and dyspnea is often paralleled with increased cardiac injury biomarkers. However, an atypical clinical presentation of cardiac injury is often blunted by the symptoms of lung infection. It should be carefully identified to take action before the condition rapidly deteriorates.

\section{Prospect and challenges}

Cardiovascular diseases in patients with COVID-19 are accountable for the poor prognosis especially in $>50$-year old adults with underlying
CVD. Recently emerging evidence helps explain the structural features of the SARS-CoV-2, the way it interacts with human cells and its infection ability [4, 47-49], however, why SARS-CoV-2 has a much more efficient transmission and spreads much faster than SARS-CoV though they belong to the same species is not known. As for CVDs, the exact mechanism by which SARS-CoV-2 results in dysfunction of the cardiovascular system, the relationship of SARS-CoV-2, ACE2 and ACEI/ /ARB remains unknown. Second, it is essential to develop targeted antiviral drugs and vaccines with strict standards using standard clinical-trial parameters. Third, there are some concerns that when SARS-CoV-2 is widely circulating, SARS-CoV-2 may result in an outbreak to overwhelm the healthcare systems. Fourth, there are many silent coronavirus spreaders who are infected by SARS-CoV-2 but show no symptoms. Less is known about their infectiousness and prognosis. Fifth, as COVID-19 is rapidly spreading across the world, enormous and scarce medical resources are allocated to combat the unprecedented pandemic. These efforts aiming to contain the circulation of SARS-CoV-2 will inevitably lead to absolute scarcity in other fields. Numerous pleas have been received from patients with underlying CVD, diabetes mellitus, and other conditions for routine clinical services such as elective PCI, ablation of cardiac arrhythmias, or cardiac rehabilitation. When most if not all of those patients take a back seat during the unpredictable COVID-19 pandemic, they will be critically affected and considered as a high-risk population of severe and critical conditions prone to SARS-CoV-2 infection. Therefore, preparedness for maximizing scarce medical resources across all patients is essential.

\section{Acknowledgements}

All authors thank Dr. Lv Liu for sharing their valuable experience in managing patients with COVID-19 in Wuhan, China; and Dr. Changcong Cui and Danjun Zhu for helpful comments and discussion on the manuscript. This work was supported by the Clinical Research Award of the First Affiliated Hospital of Xi'an Jiaotong University, China (XJTU1AF-CRF-2018-015).

\section{Conflict of interest: None declared}

\section{References}

1. Zhou P, Yang XL, Wang XG, et al. A pneumonia outbreak associated with a new coronavirus of probable bat origin. Nature. 2020; 579(7798): 270-273, doi: 10.1038/s41586-020-2012-7. 
2. Wölfel R, Corman VM, Guggemos W, et al. Virological assessment of hospitalized patients with COVID-2019. Nature. 2020; 581(7809): 465-469, doi: 10.1038/s41586-020-2196-x, indexed in Pubmed: 32235945.

3. Vaduganathan M, Vardeny O, Michel T, et al. Renin-angiotensin-aldosterone system inhibitors in patients with COVID-19. N Engl J Med. 2020; 382(17): 1653-1659, doi: 10.1056/NEJMsr2005760, indexed in Pubmed: 32227760.

4. Letko M, Marzi A, Munster V. Functional assessment of cell entry and receptor usage for SARS-CoV-2 and other lineage B betacoronaviruses. Nat Microbiol. 2020; 5(4): 562-569, doi: 10.1038/s41564-020-0688-y, indexed in Pubmed: 32094589.

5. Grasselli G, Zangrillo A, Zanella A, et al. COVID-19 Lombardy ICU Network. Baseline characteristics and outcomes of 1591 patients infected with SARS-CoV-2 admitted to icus of the lombardy region, Italy. JAMA. 2020; 323(16): 1574-1581, doi: 10.1001/jama.2020.5394, indexed in Pubmed: 32250385.

6. Zhou F, Yu T, Du R, et al. Clinical course and risk factors for mortality of adult inpatients with COVID-19 in Wuhan, China: a retrospective cohort study. Lancet. 2020; 395(10229): 1054-1062, doi: 10.1016/S0140-6736(20)30566-3, indexed in Pubmed: 32171076 .

7. Huang C, Wang Y, Li X, et al. Clinical features of patients infected with 2019 novel coronavirus in Wuhan, China. Lancet. 2020; 395(10223): 497-506, doi: 10.1016/S0140-6736(20)30183-5, indexed in Pubmed: 31986264.

8. Bian XW. Autopsy of COVID-19 victims in China. National Science Review. 2020; nwaa123, doi: 10.1093/nsr/nwaa123.

9. Wu Z, McGoogan JM. Characteristics of and important lessons from the coronavirus disease 2019 (COVID-19) outbreak in china: summary of a report of 72314 cases from the chinese center for disease control and prevention. JAMA. 2020; 323(13): 1239-1242, doi: 10.1001/jama.2020.2648, indexed in Pubmed: 32091533.

10. Shi S, Qin Mu, Shen Bo, et al. Association of Cardiac Injury With Mortality in Hospitalized Patients With COVID-19 in Wuhan, China. JAMA Cardiol. 2020; 5(7): 802-810, doi: 10.1001/jamacardio.2020.0950, indexed in Pubmed: 32211816.

11. Li JW, Han TW, Woodward M, et al. The impact of 2019 novel coronavirus on heart injury: a systematic review and Meta-analysis. Prog Cardiovasc Dis. 2020; 63(4): 518-524, doi: 10.1016/j. pcad.2020.04.008, indexed in Pubmed: 32305557.

12. Lippi G, Lavie CJ, Sanchis-Gomar F. Cardiac troponin I in patients with coronavirus disease 2019 (COVID-19): Evidence from a meta-analysis. Prog Cardiovasc Dis. 2020; 63(3): 390-391, doi: 10.1016/j.pcad.2020.03.001, indexed in Pubmed: 32169400.

13. Xu Z, Shi L, Wang Y, et al. Pathological findings of COVID-19 associated with acute respiratory distress syndrome. Lancet Respir Med. 2020; 8(4): 420-422, doi: 10.1016/s2213-2600(20)30076-x.

14. Inciardi RM, Lupi L, Zaccone G, et al. Cardiac Involvement in a Patient With Coronavirus Disease 2019 (COVID-19). JAMA Cardiol. 2020; 5(7): 819-824, doi: 10.1001/jamacardio.2020.1096, indexed in Pubmed: 32219357.

15. $\mathrm{Hu} \mathrm{H}, \mathrm{Ma} \mathrm{F}$, Wei X, et al. Coronavirus fulminant myocarditis saved with glucocorticoid and human immunoglobulin. Eur Heart J. 2020 [Epub ahead of print], doi: 10.1093/eurheartj/ehaa190, indexed in Pubmed: 32176300.

16. Deng Q, Hu Bo, Zhang Y, et al. Suspected myocardial injury in patients with COVID-19: Evidence from front-line clinical observation in Wuhan, China. Int J Cardiol. 2020; 311: 116-121, doi: 10.1016/j.ijcard.2020.03.087, indexed in Pubmed: 32291207.
17. Wang C, Xie J, Zhao L, et al. Aveolar macrophage activation and cytokine storm in the pathogenesis of severe COVID-19. 2020, doi: 10.21203/rs.3.rs-19346/v1.

18. Chen N, Zhou M, Dong X, et al. Epidemiological and clinical characteristics of 99 cases of 2019 novel coronavirus pneumonia in Wuhan, China: a descriptive study. Lancet. 2020; 395(10223): 507-513, doi: 10.1016/s0140-6736(20)30211-7.

19. National Center for Gerontology/ National Clinical Research Center for Geriatric Disorders, Cardiovascular Branch of Chinese Geriatrics Society, Imaging Group of Cardiovascular Department, Beijing Medical Association Expert Recommendations for Clinical Management of Myocardial Injury Associated With Coronavirus Disease 2019 (First Edition). Chinese Circulation J, doi: 10.3969/j.issn.1000-3614.2020.04.000.

20. Bangalore S, Sharma A, Slotwiner A, et al. ST-Segment elevation in patients with COVID-19 - a case series. $\mathrm{N}$ Engl J Med. 2020; 382(25): 2478-2480, doi: 10.1056/NEJMc2009020, indexed in Pubmed: 32302081.

21. Chorin E, Dai M, Shulman E, et al. The QT interval in patients with COVID-19 treated with hydroxychloroquine and azithromycin. Nat Med. 2020; 26(6): 808-809, doi: 10.1038/s41591-0200888-2, indexed in Pubmed: 32488217.

22. Li Y, Liu T, Liu M, et al. Electrocardiogramic abnormities in patients with COVID-19. Chin J Arhythmia (in Chinese). 2020, doi: 10.3760/cma.j.cn.113859-20200302-20200044.

23. Mitra RL, Greenstein SA, Epstein LM. An algorithm for managing QT prolongation in coronavirus disease 2019 (COVID-19) patients treated with either chloroquine or hydroxychloroquine in conjunction with azithromycin: Possible benefits of intravenous lidocaine. Heart Rhythm Case Rep. 2020; 6(5): 244-248, doi: 10.1016/j.hrcr.2020.03.016, indexed in Pubmed: 32363145.

24. Gabriels J, Saleh M, Chang D, et al. Inpatient use of mobile continuous telemetry for COVID-19 patients treated with hydroxychloroquine and azithromycin. Heart Rhythm Case Rep. 2020; 6(5): 241-243, doi: 10.1016/j.hrcr.2020.03.017, indexed in Pubmed: 32363144.

25. Rosenberg ES, Dufort EM, Udo T, et al. Association of treatment with hydroxychloroquine or azithromycin with in-hospital mortality in patients with COVID-19 in New York state. JAMA. 2020; 323(24): 2493-2502, doi: 10.1001/jama.2020.8630, indexed in Pubmed: 32392282.

26. Peng YD, Meng K, Guan HQ, et al. [Clinical characteristics and outcomes of 112 cardiovascular disease patients infected by 2019-nCoV]. Zhonghua Xin Xue Guan Bing Za Zhi. 2020; 48(6): 450-455, doi: 10.3760/cma.j.cn112148-20200220-00105, indexed in Pubmed: 32120458.

27. Han Y, Zeng H, Jiang H, et al. CSC expert consensus on principles of clinical management of patients with severe emergent cardiovascular diseases during the COVID-19 epidemic. Circulation. 2020; 141(20): e810-e816, doi: 10.1161/CIRCULATIONAHA.120.047011, indexed in Pubmed: 32216640.

28. Jing ZC, Zhu HD, Yan XW, et al. Recommendations from the Peking Union Medical College Hospital for the management of acute myocardial infarction during the COVID-19 outbreak. Eur Heart J. 2020; 41(19): 1791-1794, doi: 10.1093/eurheartj/ ehaa258, indexed in Pubmed: 32232396.

29. Clerkin KJ, Fried JA, Raikhelkar J, et al. COVID-19 and cardiovascular disease. Circulation. 2020; 141(20): 1648-1655, doi: 10.1161/CIRCULATIONAHA.120.046941, indexed in Pubmed: 32200663. 
30. Zheng HY, Zhang Mi, Yang CX, et al. Elevated exhaustion levels and reduced functional diversity of $\mathrm{T}$ cells in peripheral blood may predict severe progression in COVID-19 patients. Cell Mol Immunol. 2020; 17(5): 541-543, doi: 10.1038/s41423-020-0401-3, indexed in Pubmed: 32203186.

31. Thevarajan I, Nguyen THO, Koutsakos M, et al. Breadth of concomitant immune responses prior to patient recovery: a case report of non-severe COVID-19. Nat Med. 2020; 26(4): 453-455, doi: 10.1038/s41591-020-0819-2, indexed in Pubmed: 32284614.

32. Kuba K, Imai Y, Rao S, et al. A crucial role of angiotensin converting enzyme 2 (ACE2) in SARS coronavirus-induced lung injury. Nat Med. 2005; 11(8): 875-879, doi: 10.1038/nm1267, indexed in Pubmed: 16007097.

33. Yang $\mathrm{P}, \mathrm{Gu} \mathrm{H}, \mathrm{Zhao} \mathrm{Z}$, et al. Angiotensin-converting enzyme 2 (ACE2) mediates influenza H7N9 virus-induced acute lung injury. Sci Rep. 2014; 4: 7027, doi: 10.1038/srep07027, indexed in Pubmed: 25391767.

34. Oudit GY, Kassiri Z, Jiang C, et al. SARS-coronavirus modulation of myocardial ACE2 expression and inflammation in patients with SARS. Eur J Clin Invest. 2009; 39(7): 618-625, doi: 10.1111/j.1365-2362.2009.02153.x, indexed in Pubmed: 19453650.

35. Wrapp D, Wang N, Corbett KS, et al. Cryo-EM structure of the 2019-nCoV spike in the prefusion conformation. Science. 2020; 367(6483): 1260-1263, doi: 10.1126/science.abb2507, indexed in Pubmed: 32075877.

36. Varga Z, Flammer AJ, Steiger P, et al. Endothelial cell infection and endotheliitis in COVID-19. Lancet. 2020; 395(10234): 1417-1418, doi: 10.1016/S0140-6736(20)30937-5, indexed in Pubmed: 32325026.

37. Gautret P, Lagier JC, Parola P, et al. Hydroxychloroquine and azithromycin as a treatment of COVID-19: results of an openlabel non-randomized clinical trial. Int J Antimicrobial Agents. 2020: 105949, doi: 10.1016/j.ijantimicag.2020.105949.

38. Joyce E, Fabre A, Mahon N. Hydroxychloroquine cardiotoxicity presenting as a rapidly evolving biventricular cardiomyopathy: key diagnostic features and literature review. Eur Heart J Acute Cardiovasc Care. 2013; 2(1): 77-83, doi: 10.1177/2048872612471215, indexed in Pubmed: 24062937.

39. Cao B, Wang Y, Wen D, et al. A trial of lopinavir-ritonavir in adults hospitalized with severe COVID-19. N Engl J Med. 2020;
382(19): 1787-1799, doi: 10.1056/NEJMoa2001282, indexed in Pubmed: 32187464.

40. Raoult D. Hydroxychloroquine-COVID-19 study did not meet publishing society's, expected standard. 2020.

41. Molina JM, Delaugerre C, Le Goff J, et al. No evidence of rapid antiviral clearance or clinical benefit with the combination of hydroxychloroquine and azithromycin in patients with severe COVID-19 infection. Med Mal Infect. 2020; 50(4): 384, doi: 10.1016/j.medmal.2020.03.006, indexed in Pubmed: 32240719.

42. Guan L, Zhou L, Zhang J, et al. More awareness is needed for severe acute respiratory syndrome coronavirus 2019 transmission through exhaled air during non-invasive respiratory support: experience from China. Eur Respir J. 2020; 55(3), doi: 10.1183/13993003.00352-2020, indexed in Pubmed: 32198275.

43. Russell C, Millar J, Baillie J. Clinical evidence does not support corticosteroid treatment for 2019-nCoV lung injury. Lancet. 2020; 395(10223): 473-475, doi: 10.1016/s0140-6736(20)30317-2.

44. Yao H, Chen JH, Xu YF. Patients with mental health disorders in the COVID-19 epidemic. Lancet Psychiatry. 2020; 7(4): e21, doi: 10.1016/s2215-0366(20)30090-0.

45. Brooks S, Webster R, Smith L, et al. The psychological impact of quarantine and how to reduce it: rapid review of the evidence. Lancet. 2020; 395(10227): 912-920, doi: 10.1016/s01406736(20)30460-8.

46. Jiménez-Pavón D, Carbonell-Baeza A, Lavie CJ. Physical exercise as therapy to fight against the mental and physical consequences of COVID-19 quarantine: Special focus in older people. Prog Cardiovasc Dis. 2020; 63(3): 386-388, doi: 10.1016/j. pcad.2020.03.009, indexed in Pubmed: 32220590.

47. Walls AC, Park YJ, Tortorici MA, et al. Structure, function, and antigenicity of the SARS-CoV-2 spike glycoprotein. Cell. 2020; 181(2): 281-292.e6, doi: 10.1016/j.cell.2020.02.058, indexed in Pubmed: 32155444.

48. Lan J, Ge J, Yu J, et al. Structure of the SARS-CoV-2 spike receptor-binding domain bound to the ACE2 receptor. Nature. 2020; 581(7807): 215-220, doi: 10.1038/s41586-020-2180-5, indexed in Pubmed: 32225176.

49. Lam TTY, Jia Na, Zhang YW, et al. Identifying SARS-CoV-2-related coronaviruses in Malayan pangolins. Nature. 2020; 583(7815): 282-285, doi: 10.1038/s41586-020-2169-0, indexed in Pubmed: 32218527 . 